\title{
Evaluation of different culture media to support in vitro growth and biofilm formation of bacterial vaginosis- associated anaerobes
}

\author{
Aliona S Rosca ${ }^{1}$, Joana Castro ${ }^{1}$, Nuno Cerca ${ }^{\text {Corresp. } 1}$ \\ ${ }^{1}$ Laboratory of Research in Biofilms Rosário Oliveira - Centre of Biological Engineering, University of Minho, Braga, Portugal \\ Corresponding Author: Nuno Cerca \\ Email address: nunocerca@ceb.uminho.pt
}

Background. Bacterial vaginosis (BV) is one of the most common vaginal infections worldwide. It is associated with the presence of a dense polymicrobial biofilm on the vaginal epithelium, formed mainly by Gardnerella species. The biofilm also contains other anaerobic species, but little is known about their role in BV development. Aim. To evaluate the influence of different culture media on the planktonic and biofilm growth of six cultivable anaerobes frequently associated with BV, namely Gardnerella sp., Atopobium vaginae, Lactobacillus iners, Mobiluncus curtisii, Peptostreptococcus anaerobius, and Prevotella bivia. Methods. A total of nine different culture media compositions, including commercially available and chemically defined media simulating genital tract secretions, were tested in this study. Planktonic cultures and biofilms were grown under anaerobic conditions ( $10 \%$ carbon dioxide, $10 \%$ helium, and $80 \%$ nitrogen). Planktonic growth was assessed by optical density measurements, and biofilm formation was quantified by crystal violet staining. Results. Significant planktonic growth was observed for Gardnerella sp., A. vaginae, and L. iners in New York City III broth, with or without ascorbic acid supplementation. Biofilm quantification showed high in vitro biofilm growth for Gardnerella sp., $P$. anaerobius, and $P$. bivia in almost all culture media excluding Brucella broth. Contrary, only New York City III broth was able to promote biofilm formation for A. vaginae, L. iners, and M. curtisii. Conclusions. Our data demonstrate that New York City III broth relative to the other tested media is the most conducive for future studies addressing polymicrobial biofilms development as this culture medium allowed the formation of significant levels of single-species biofilms. 
1 Evaluation of different culture media to support in vitro

2 growth and biofilm formation of bacterial vaginosis-

3 associated anaerobes

4 Aliona S Rosca, Joana Castro, Nuno Cerca

5 Laboratory of Research in Biofilms Rosário Oliveira - Centre of Biological Engineering,

6 University of Minho, Braga, Portugal

7

8 Corresponding Author:

9 Nuno Cerca

10 Campus de Gualtar, Braga, 4710-057, Portugal

11 Email address: nunocerca@,ceb.uminho.pt

12

13

14

15

16

17

18

19

20

Abstract (257/ 500 words)

Manuscript (3083 words)

21

22

23

24

25

26

27

28

29

30

Peer] reviewing PDF | (2020:04:47424:1:1:NEW 31 Jul 2020) 
31 Abstract

32 Background. Bacterial vaginosis (BV) is one of the most common vaginal infections worldwide.

33 It is associated with the presence of a dense polymicrobial biofilm on the vaginal epithelium,

34 formed mainly by Gardnerella species. The biofilm also contains other anaerobic species, but

35 little is known about their role in BV development.

36 Aim. To evaluate the influence of different culture media on the planktonic and biofilm growth

37 of six cultivable anaerobes frequently associated with BV, namely Gardnerella sp., Atopobium vaginae, Lactobacillus iners, Mobiluncus curtisii, Peptostreptococcus anaerobius, and Prevotella bivia.

40 Methods. A total of nine different culture media compositions, including commercially available and chemically defined media simulating genital tract secretions, were tested in this study. Planktonic cultures and biofilms were grown under anaerobic conditions (10\% carbon dioxide, $10 \%$ helium, and $80 \%$ nitrogen). Planktonic growth was assessed by optical density measurements, and biofilm formation was quantified by crystal violet staining.

Results. Significant planktonic growth was observed for Gardnerella sp., A. vaginae, and $L$. iners in New York City III broth, with or without ascorbic acid supplementation. Biofilm quantification showed high in vitro biofilm growth for Gardnerella sp., P. anaerobius, and $P$. bivia in almost all culture media excluding Brucella broth. Contrary, only New York City III broth was able to promote biofilm formation for A. vaginae, L. iners, and M. curtisii.

Conclusions. Our data demonstrate that New York City III broth relative to the other tested media is the most conducive for future studies addressing polymicrobial biofilms development as this culture medium allowed the formation of significant levels of single-species biofilms. 
58

59

60

61

62

63

64

65

66

67

68

69

70

71

\section{Introduction}

Bacterial vaginosis (BV) is the worldwide leading bacterial vaginal infection commonly recognized in women of all ethnicities between menarche and menopause (Beamer et al., 2017; Javed et al., 2019). BV is often characterized by a decrease of beneficial vaginal bacteria, mainly hydrogen peroxide and lactic acid-producing Lactobacillus species, and by an increase of anaerobic pathogens (Marrazzo, 2011; Schwebke, 2000). The most prominent of these is Gardnerella vaginalis, a facultative anaerobe usually found embedded in a polymicrobial biofilm (Swidsinski et al., 2014). It is important to mention that an emended description of G. vaginalis was recently proposed with the delineation of 13 genomic species within the genus Gardnerella (Vaneechoutte et al., 2019). Of these 13 species, three were officially described (G. leopoldii, G. piotii, and G. swidsinskii) and G. vaginalis was amended. Following this renewed taxonomy of the genus Gardnerella, in this article, the term Gardnerella spp. will be used to address previous publications, since we cannot rule out the fact that other Gardnerella species were involved.

According to the current hypothesis for BV pathogenesis, Gardnerella spp. initiate the formation of the biofilm on vaginal epithelial cells and become a scaffolding to which other BV-associated species thereafter can attach (Muzny et al., 2019). One of the species that is often found associated with Gardnerella spp. biofilms is Atopobium vaginae (Swidsinski et al., 2005). Under specific in vitro conditions, $G$. vaginalis enhances culturability of $A$. vaginae (Castro et al., 2020) and it has been suggested that the in vivo detection of both bacteria is a strong indicator of $\mathrm{BV}$ development (Bradshaw et al., 2006; Hardy et al., 2016, 2015; Muzny et al., 2019). However, the BV biofilm is often populated by many other facultative or strict anaerobes, but very little is known about their role in BV development. As such, more studies are needed to address the interactions between these species. One issue facing researchers that work with BV-associated species is that most species are uncultivable or fastidious (Diop et al., 2019; Fredricks et al., 2007, 2005; Srinivasan et al., 2016). Furthermore, in vitro biofilm formation requirements are often different from planktonic growth (Alves et al., 2014). Considering the increased focus on biofilm-associated infections and the demand for finding novel treatment approaches (Falconi-McCahill, 2019), the current study was undertaken aiming to evaluate the effects of nine different culture media on the planktonic growth and biofilm formation of cultivable anaerobes frequently found in BV, namely Gardnerella sp., A. vaginae, Mobiluncus curtisii, 
Peptostreptococcus anaerobius, and Prevotella bivia (Diop et al., 2019; Onderdonk et al., 2016). Importantly, Lactobacillus iners was also included in this study as this species plays a controversial role in the vaginal microenvironment, being detected in the vaginal microbiota of both healthy (Anukam et al., 2006; Ravel et al., 2011) and women with BV (Rampersaud et al., 2011). Furthermore, L. iners has been often identified in the intermediate vaginal microbiota (i.e. between normal and BV microbiota) (Jakobsson and Forsum, 2007; Petrova et al., 2015) and also dominates the microbiota after treatment of BV (Ferris et al., 2007). Also, this microorganism has complex nutritional requirements, being thus, not easy to work with in vitro (Vaneechoutte, 2017).

As such, the main goal of this study was to explore growth conditions optimal for using in future in vitro multi-species biofilm model, with the aim to better analyse BV multi-species interactions and their impact on BV development.

\section{Materials \& Methods}

\section{Bacterial species and growth conditions}

Six cultivable bacterial species associated with BV were used in the current study, namely Gardnerella sp., A. vaginae, L. iners, M. curtisii, P. anaerobius, and P. bivia (Table 1). These species were preserved frozen in Brain Heart Infusion broth (BHI) (Liofilchem, Italy) with 23\% (v/v) glycerol (Panreac, Spain) at $-80^{\circ} \mathrm{C}$. Each species was inoculated from the $-80^{\circ} \mathrm{C}$ bacterial stock on plates containing Columbia Blood Agar medium (CBA) (Oxoid, UK) supplemented with $5 \%(\mathrm{v} / \mathrm{v})$ defibrinated horse blood (Oxoid, UK) and incubated at $37^{\circ} \mathrm{C}$ under anaerobic conditions [controlled atmosphere composed of 10\% carbon dioxide $\left(\mathrm{CO}_{2}\right), 10 \%$ helium, and $80 \%$ nitrogen generated by a cylinder (Air Liquid, Algés, Portugal) coupled to an anaerobic incubator (Plas-Labs, Lansing, MI, USA)] for 2-4 days. For planktonic and biofilm assays, Brain heart infusion broth supplemented with yeast extract, starch, and gelatine (sBHI), Brucella broth supplemented with hemin and vitamin $\mathrm{K}_{1}$ solutions (BHV), New York City III broth supplemented with 10\% inactivated horse serum (NYC), Schaedler broth (SB), and a medium simulating genital tract secretions (mGTS) were used as culture media with the mentioned composition, but also supplemented with $0.1 \%$ (w/v) L-ascorbic acid (Sigma-Aldrich, UK), excepting mGTS which already contains L-ascorbic acid. The addition of L-ascorbic acid to the 
118 culture media was designated with the abbreviation "Aa", added at the end of each medium's

119 name mentioned above (e.g. sBHI supplemented with L-ascorbic acid became sBHI.Aa). The

120 detailed information about each tested medium is presented in Table 2. In order to prepare hemin

121 solution, $0.5 \mathrm{~g}$ of hemin was dissolved in $10 \mathrm{~mL}$ of $1 \mathrm{~N} \mathrm{NaOH}$ and afterwards distilled water was

122 added to reach the volume of $100 \mathrm{~mL}$. Further, vitamin $\mathrm{K}_{1}$ solution was prepared by adding

$1230.025 \mathrm{~mL}$ of vitamin $\mathrm{K}_{1}$ stock solution to $4.975 \mathrm{~mL}$ of $95 \%$ ethanol. The prepared solutions of

124 hemin and vitamin $\mathrm{K}_{1}$ were used with a concentration of $0.0005 \%(\mathrm{w} / \mathrm{v})$ and $0.0001 \%(\mathrm{w} / \mathrm{v})$,

125 respectively. In mGTS, Part III of this medium is a vitamin mixture, Sigma K3129, from Sigma-

126 Aldrich (UK) with the stock solution of $100 \mathrm{X}$ that was used at a concentration of $0.5 \%(\mathrm{v} / \mathrm{v})$.

127

128 Planktonic growth assessment

129 For the evaluation of planktonic growth, the inoculums were prepared by transferring fresh

130 bacterial colonies from CBA plates to $8 \mathrm{~mL}$ of each culture medium described above. The

131 obtained bacterial suspensions were adjusted by optical density (OD) at $620 \mathrm{~nm}$ to $0.10 \pm 0.05$

132 (Biochrom EZ Read 800 Plus, UK) and equally distributed in 2 sterile $15 \mathrm{~mL}$ falcon tubes

133 (Orange Scientific, Belgium) which were further incubated at $37^{\circ} \mathrm{C}$ under anaerobic conditions

134 for $48 \mathrm{~h}$, as described above. Afterwards, planktonic growth was assessed by $\mathrm{OD}_{620 \mathrm{~nm}}$. Growth

135 was normalized as a fold difference between the final $\mathrm{OD}_{620 \mathrm{~nm}}$ and the starting $\mathrm{OD}$ (at time $0 \mathrm{~h}$ ).

136 The assays were repeated at least three times on separate days, with four technical replicates

137 considered each time.

138

139

Biofilm formation and biomass quantification

140

Single-species biofilms of each tested species were initiated by inoculating bacterial suspensions of $48 \mathrm{~h}$ cultures adjusted to an $\mathrm{OD}_{620 \mathrm{~nm}}$ of $0.10 \pm 0.05$ in sterile 96-well tissue culture plates (Orange Scientific, Belgium) and incubated for $72 \mathrm{~h}$, at $37{ }^{\circ} \mathrm{C}$ under anaerobic conditions. To quantify the biofilm biomass, we used the crystal violet (CV) method, which is the most

144 frequently employed approach for this purpose (Azeredo et al., 2017; Peeters et al., 2008). In brief, following $72 \mathrm{~h}$ of incubation, the biofilms were washed once with $0.9 \%(\mathrm{w} / \mathrm{v})$ sodium chloride and allowed to air dry. After, the biofilms were fixed with $100 \%(\mathrm{v} / \mathrm{v})$ methanol

147 (Thermo Fisher Scientific) for $20 \mathrm{~min}$, and then stained with CV solution 1\% (v/v) (Merck, Darmstadt, Germany) for 20 min. Subsequently, each well was washed twice with 1\% (v/v) 
149 phosphate-buffered saline, and the bound CV was released with 33\% (v/v) acetic acid (Thermo

150 Fisher Scientific, Lenexa, KS). To assess the biomass, the OD of the resulting solution was

151 measured at $595 \mathrm{~nm}$. Biofilm experiments were repeated at least three times with eight technical

152 replicates.

153

154 Statistical analysis

155 The data were analysed using the statistical package GraphPad Prism version 6 (La Jolla, CA, 156 USA) by one-way ANOVA (Dunnett's and Tukey's multiple comparison tests) and two-way

157 ANOVA (Sidak's multiple comparisons test). Values with a $p<0.05$ and $p<0.01$ were

158 considered statistically significant.

159

160

161

162

163

164

165

166

167

168

169

170

171

172

173

174

175

176

177

178

\section{Results}

\section{Planktonic growth assays}

As shown in Fig. 1, BV-associated anaerobes had variable ability to grow planktonically in the tested culture media. Accordingly, P. anaerobius and P. bivia had higher metabolic flexibility and were able to grow in most of the tested media, while $M$. curtisii had more restrictive growth requirements and presented low levels of growth in all of them. Interestingly, NYC broth showed high levels of planktonic growth for the tested species, being overpassed only by NYC.Aa for $L$. iners, SB and SB.Aa for P. anaerobius, and by sBHI.Aa for P. bivia. The mGTS supported very low levels of bacterial growth, with only Gardnerella sp., P. anaerobius, and P. bivia showing moderate levels of growth.

Since it was previously shown that L-ascorbic acid could enhance the growth of several anaerobic bacteria, including A. vaginae, Finegoldia magna, Fusobacterium necrophorum, Prevotella nigrescens, Ruminococcus gnavus, and Solobacterium moorei (La Scola et al., 2014), we repeated the experiments with media supplemented with $0.1 \%(\mathrm{w} / \mathrm{v}) \mathrm{L}$-ascorbic acid. However, contrary to what was described before, the addition of $0.1 \%(\mathrm{w} / \mathrm{v}) \mathrm{L}$-ascorbic acid had a very variable effect on bacterial growth, with only $33.3 \%$ ( $n=8$; cut-off $\geq 1.25$ - fold change) of the total combinations tested yielding a significant increase in bacterial growth, while in $4.17 \%$ $(n=1$; cut-off $<0.75$-fold change) an inhibition of the growth was observed. In most of the tested 
179

180

181

182

183

184

185

186

187

188

189

190

191

192

193

194

195

196

197

198

199

200

201

202

203

204

205

206

207

combinations ( $\mathrm{n}=15 ; 0.75 \leq$ fold change $<1.25)$, no effect was observed (Table S1). The most notable case was observed for $P$. bivia growth in SB, in which L-ascorbic acid increased almost seven-fold the growth rate.

\section{Biofilm assays}

Not surprisingly, we observed that similar to planktonic growth, biofilm formation was also strongly affected by the culture media composition, as depicted in Fig. 2. Importantly, there was not a direct relationship between higher planktonic growth and higher biofilm formation, which further confirms that the requirements for biofilm formation are distinct than the requirements for planktonic growth, as showed before for many other bacterial species (Alves et al., 2014; Heffernan et al., 2009; Ripolles-Avila et al., 2018; Wijesinghe et al., 2019). Further differences between biofilm formation and planktonic growth were observed when adding L-ascorbic acid to the growth media, with $20.8 \%(n=5)$ of the tested combinations species/ growth medium resulting in a statistically significant decrease in the biofilm-forming capacity $(p<0.05)$ and $37.5 \%(\mathrm{n}=9)$ of the situations also presenting a visible biofilm reduction, however not statistically significant as can be seen in Table S2. Moreover, the addition of L-ascorbic acid to the culture media did not promote significantly increased biofilm formation in any of the combinations tested (Fig. 2).

\section{Discussion}

Despite the fact that BV is an increasingly important health problem, there is a lack of studies addressing multi-species interactions that might occur during BV and their role in the development of this infection. Most attempts to understand the microbiology behind BV have been focused mainly on Gardnerella spp., perhaps because this species has long been associated with BV development (Gardner and Dukes, 1955; Swidsinski et al., 2005) and it has been now hypothesized that this microorganism is the initial colonizer of the vaginal epithelium, being able to establish an early biofilm structure to which other BV-associated species can attach (Muzny et al., 2019). However, the role of these species in the development and progress of BV is still poorly understood and therefore, more studies are needed to unravel this matter. 
208 We showed before that BV-associated species had different abilities to grow as biofilms, and this 209 was strongly dependent on the growth media (Alves et al., 2014). As such, the first step in 210 facilitating BV multi-species biofilm studies is to determine optimal culture medium conditions 211 suitable for multiple BV-associates species, considering to further investigate the interactions 212 that might exist between them in BV multi-species biofilms and their implications in BV 213 process.

214 Although sBHI has been widely used as a medium that supports Gardnerella spp. growth 215 (Algburi et al., 2015; Gottschick et al., 2016; Harwich et al., 2010; Machado et al., 2015; 216 Patterson et al., 2010; Turovskiy et al., 2012; Weeks et al., 2019), it did not facilitate the 217 planktonic growth or biofilm formation for some of the tested species, including A. vaginae, L.

218 iners, and M. curtisii. The same was observed for these three species in SB medium, even though 219 the manufacturer describes it as a medium suitable for the cultivation of anaerobic 220 microorganisms, providing them an important amount of amino acids, nitrogen, vitamins as well 221 as the energy necessary for growth. In an early study, after evaluating nine broth media in varied $222 \mathrm{CO}_{2}$ atmospheres for their ability to support the growth of anaerobic bacteria including 223 Bacteroides fragilis subspecies fragilis, Peptostreptococcus CDC group 2, Eubacterium 224 alactolyticum, and Clostridium perfringens, Stalons and colleagues found that SB in an 225 atmosphere of $5 \% \mathrm{CO}_{2}, 10 \%$ hydrogen, and $85 \%$ nitrogen exhibited the fastest and highest 226 growth response (Stalons et al., 1974). However, in our in vitro conditions, we obtained high 227 levels of planktonic growth only for P. anaerobius, probably because this medium is not 228 appropriate for the growth of all species of anaerobic microorganisms. Still, SB was a good 229 medium to support in vitro biofilm formation with high levels of the biomass for Gardnerella sp., P. anaerobius, and P. bivia. Interestingly, Gardnerella sp. and P. bivia showed in SB the lowest levels of planktonic growth, but the highest biofilm formation ability. As mentioned, SB is a complex medium and perhaps the presence of certain growth factors determined these two anaerobes to turn on the expression of biofilm-related genes. Another of the tested media, BHV, also described by the manufacturer as suitable for the cultivation of anaerobes, was not appropriate for the growth and biofilm formation of the tested species, with the exception of 236 planktonic growth by P. anaerobius (Fig. 1). 
237 Interestingly, NYC facilitated the planktonic growth of all tested species, despite $M$. curtisii

238 presented a very slow growth rate. Nevertheless, even $M$. curtisii was able to form high biofilm

239 biomass in this medium. In fact, together with $A$. vaginae and L. iners, significant biofilm

240 formation was only detected in NYC (Fig. 2). A particularity of NYC medium, compared to the

241 other tested media, is the presence of proteose peptone no. 3, which has been described by the

242 manufacturer as offering high nutritional benefits to fastidious anaerobic species by providing

243 the necessary amount of nitrogen, carbon, amino acids, and essential growth factors. To assess if,

244 in fact, the enhancement of biofilm formation in NYC was mainly due to the presence of

245 proteose peptone no. 3, we carried out an experiment by evaluating the biofilm-formation ability

246 of the six tested bacterial species in the original receipt of NYC versus an altered version of

247 NYC [with regular peptone from meat (Acros Organics, UK) replacing the proteose peptone no.

248 3]. Interestingly, while we did find that proteose peptone no. 3 was essential to the biofilm

249 formation by M. curtisii, no significant differences were found for the other species (Fig. S1),

250 which suggests that NYC's ability to enhance biofilm formation is not only related to the

251 presence of proteose peptone no. 3.

252 Besides the commercially available media, we also tested a chemically defined medium that

253 simulates the genital tract fluid, mGTS (Stingley et al., 2014). Since mGTS is a minimal medium

254 without rich nutrient sources, it was not surprising that the growth of the tested BV-associated

255 species was negligent or very slow in this medium. Nevertheless, biofilm formation by

256 Gardnerella sp. and P. anaerobius was significant under mGTS, further being confirmed that

257 biofilm formation requires specific conditions, different from planktonic growth.

258 We also tested another variable in our growth conditions optimization. The addition of L-

259 ascorbic acid had the advantage of reducing the oxidation potential of the growth media by

260 removing the oxygen (La Scola et al., 2014). However, the effect of adding L-ascorbic acid was

261 very variable, depending not only on the bacterial species but also on the respective growth

262 media. Nevertheless, there was a tendency to slightly or highly suppress biofilm formation.

263 Interestingly, the inhibition of biofilm formation by ascorbic acid has been described before in

264 biofilms of Bacillus subtilis, Escherichia coli, Pseudomonas aeruginosa (Pandit et al., 2017) as

265 well as of methicillin-resistant Staphylococcus aureus (Mirani et al., 2018). It should be noted

266 that at higher concentrations, L-ascorbic acid has been reported as a possible adjuvant for 
267 antibiotic treatment of $\mathrm{BV}$, playing a role in maintaining a low vaginal $\mathrm{pH}$, which favours the

268 recolonization of the vaginal environment with lactic acid-producing bacteria, decreasing,

269 thereby, the risk of BV recurrence (Krasnopolsky et al., 2013; Polatti et al., 2006). Our data

270 further expand these previous findings by demonstrating that, while sometimes favouring

271 planktonic growth, L-ascorbic acid often impairs biofilm formation.

272 A limitation of this study was the fact that we only tested a yet unidentified Gardnerella sp.

273 isolate, but at least 3 new species have been recently reported. Previously, we assessed biofilm

274 formation by 7 clinical isolates from BV-women and 7 from healthy microbiota and found no

275 significant differences between the ability to form biofilms by the 2 groups, using different

276 growth media (Castro et al., 2015). We now know that from those 14 isolates, some belong to $G$.

277 vaginalis, G. leopoldii, G. piotii, and G. swidsinskii (Castro et al., 2020). As such, we

278 hypothesized that the 4 Gardnerella species would have similar biofilm formation abilities in our

279 growth medium of choice: NYC. To test this hypothesis, we selected one isolate of each species,

280 previously found to form similar biofilms in sBHI (Castro et al., 2020; Vaneechoutte et al.,

281 2019), and compared its biofilms with NYC medium. As shown in Fig. S2, all the tested species

282 had a similar biofilm-formation ability as compared to Gardnerella sp. UM241, with G. leopoldii

283 showing a slight decrease in biomass, but well within the expected variation found in different

284 Gardnerella strains (Castro et al., 2015).

285

\section{Conclusions}

287 Overall, our work has shed new light on the optimal conditions required for in vitro growth and biofilm formation of bacteria associated with BV. Although we tested nine different growth conditions, including a medium simulating genital tract secretions (mGTS), none of them is able to account for all growth factors present in the vaginal environment, including components of the host immune system, that are known to interfere in bacterial growth (Castro et al., 2018). Nevertheless, this work highlighted that under the appropriate in vitro conditions, some of the most common species found in BV can form single-species biofilms, contrary to what was shown before (Castro et al., 2020; Patterson et al., 2010). NYC medium revealed to be an ideal candidate for future studies addressing multi-species biofilm formation since this growth 
297

298

299

300

301

302

303

304

305

306

307

308

309

310

311

312

313

314

315

316

317

318

319

320

321

322

323

324

325

326

interactions that occur during BV development is crucial for the development of novel antimicrobial strategies, and future work will help to clarify some of these crucial interactions in multi-species biofilms.

\section{References}

Algburi, A., Volski, A., Chikindas, M.L., 2015. Natural antimicrobials subtilosin and lauramide arginine ethyl ester (LAE) synergize with conventional antibiotics clindamycin and metronidazole against biofilms of Gardnerella vaginalis but not against biofilms of healthy vaginal lactobacilli. Pathog. Dis. 73, ftv018. https://doi.org/10.1093/femspd/ftv018

Alves, P., Castro, J., Sousa, C., Cereija, T.B., Cerca, N., 2014. Gardnerella vaginalis outcompetes 29 other bacterial species isolated from patients with bacterial vaginosis, using in an in vitro biofilm formation model. J. Infect. Dis. 210, 593-596. https://doi.org/10.1093/infdis/jiu131

Anukam, K.C., Osazuwa, E.O., Ahonkhai, I., Reid, G., 2006. Lactobacillus vaginal microbiota of women attending a reproductive health care service in Benin City, Nigeria. Sex. Transm. Dis. 33, 59-62. https://doi.org/10.1097/01.olq.0000175367.15559.c4

Azeredo, J., Azevedo, N.F., Briandet, R., Cerca, N., Coenye, T., Costa, A.R., Desvaux, M., Di Bonaventura, G., Hébraud, M., Jaglic, Z., Kačániová, M., Knøchel, S., Lourenço, A., Mergulhão, F., Meyer, R.L., Nychas, G., Simões, M., Tresse, O., Sternberg, C., 2017. Critical review on biofilm methods. Crit. Rev. Microbiol. 43, 313-351. https://doi.org/10.1080/1040841X.2016.1208146

Beamer, M.A., Austin, M.N., Avolia, H.A., Meyn, L.A., Bunge, K.E., Hillier, S.L., 2017. Bacterial species colonizing the vagina of healthy women are not associated with race. Anaerobe 45, 40-43. https://doi.org/10.1016/j.anaerobe.2017.02.020

Bradshaw, C.S., Tabrizi, S.N., Fairley, C.K., Morton, a N., Rudland, E., Garland, S.M., 2006. The association of Atopobium vaginae and Gardnerella vaginalis with bacterial vaginosis and recurrence after oral metronidazole therapy. J. Infect. Dis. 194, 828-836. https://doi.org/10.1086/506621

Castro, J., Alves, P., Sousa, C., Cereija, T., França, Â., Jefferson, K.K., Cerca, N., 2015. Using an in-vitro biofilm model to assess the virulence potential of bacterial vaginosis or non- 
327

328

329

330

331

332

333

334

335

336

337

338

339

340

341

342

bacterial vaginosis Gardnerella vaginalis isolates. Sci. Rep. 5, 11640. https://doi.org/10.1038/srep11640

Castro, J., Jefferson, K., Cerca, N., 2018. Innate immune components affect growth and virulence traits of bacterial vaginosis-associated and non-BV associated Gardnerella vaginalis strains similarly. Pathog. Dis. 76, fty089. https://doi.org/10.1093/femspd/fty089

Castro, J., Rosca, A.S., Cools, P., Vaneechoutte, M., Cerca, N., 2020. Gardnerella vaginalis enhances Atopobium vaginae viability in an in vitro model. Front. Cell. Infect. Microbiol. 10, 1-9. https://doi.org/10.3389/fcimb.2020.00083

Diop, K., Dufour, J.-C., Levasseur, A., Fenollar, F., 2019. Exhaustive repertoire of human vaginal microbiota. Hum. Microbiome J. 11, 100051. https://doi.org/10.1016/j.humic.2018.11.002

Falconi-McCahill, A., 2019. Bacterial vaginosis: a clinical update with a focus on complementary and alternative therapies. J. Midwifery Womens. Health 64, 578-591. https://doi.org/10.1111/jmwh.13013

Falsen, E., Pascual, C., Sjoden, B., Ohlén, M., Collins, M.D., 1999. Phenotypic and phylogenetic characterization of a novel Lactobacillus species from human sources: description of Lactobacillus iners sp. nov. Int. J. Syst. Bacteriol. 49, 217-221. https://doi.org/10.1099/00207713-49-1-217

Ferris, M.J., Norori, J., Zozaya-hinchliffe, M., Martin, D.H., 2007. Cultivation-independent analysis of changes in bacterial vaginosis flora following metronidazole treatment. J. Clin. Microbiol. 45, 1016-1018. https://doi.org/10.1128/JCM.02085-06

Fredricks, D.N., Fiedler, T.L., Marrazzo, J.M., 2005. Molecular identification of bacteria associated with bacterial vaginosis. N. Engl. J. Med. 353, 1899-1911. https://doi.org/10.1056/NEJMoa043802

Fredricks, D.N., Fiedler, T.L., Thomas, K.K., Oakley, B.B., Marrazzo, J.M., 2007. Targeted PCR for detection of vaginal bacteria associated with bacterial vaginosis. J. Clin. Microbiol. 45, 3270-3276. https://doi.org/10.1128/JCM.01272-07

Gardner, H.L., Dukes, C.D., 1955. Haemophilus vaginalis vaginitis. Am. J. Obstet. Gynecol. 69, 962-976. https://doi.org/10.1016/0002-9378(55)90095-8

Gottschick, C., Szafranski, S.P., Kunze, B., Sztajer, H., Masur, C., Abels, C., Wagner-Döbler, I., 2016. Screening of compounds against Gardnerella vaginalis biofilms. PLoS One 11, 
358

359

360

361

362

363

364

365

366

367

368

369

370

371

372

373

374

375

376

377

378

379

380

381

382

383

384

385

386

387

388

e0154086. https://doi.org/10.1371/journal.pone.0154086

Hardy, L., Jespers, V., Abdellati, S., De Baetselier, I., Mwambarangwe, L., Musengamana, V., van de Wijgert, J., Vaneechoutte, M., Crucitti, T., 2016. A fruitful alliance: the synergy between Atopobium vaginae and Gardnerella vaginalis in bacterial vaginosis-associated biofilm. Sex. Transm. Infect. 92, 487-491. https://doi.org/10.1136/sextrans-2015-052475

Hardy, L., Jespers, V., Dahchour, N., Mwambarangwe, L., Musengamana, V., Vaneechoutte, M., Crucitti, T., 2015. Unravelling the bacterial vaginosis-associated biofilm: a multiplex Gardnerella vaginalis and Atopobium vaginae fluorescence in situ hybridization assay using peptide nucleic acid probes. PLoS One 10, e0136658. https://doi.org/10.1371/journal.pone.0136658

Harwich, M.D., Alves, J.M., Buck, G.A., Strauss, J.F., Patterson, J.L., Oki, A.T., Girerd, P.H., Jefferson, K.K., 2010. Drawing the line between commensal and pathogenic Gardnerella vaginalis through genome analysis and virulence studies. BMC Genomics 11, 375. https://doi.org/10.1186/1471-2164-11-375

Heffernan, B., Murphy, C.D., Casey, E., 2009. Comparison of planktonic and biofilm cultures of Pseudomonas fluorescens dsm 8341 cells grown on fluoroacetate. Appl. Environ. Microbiol. 75, 2899-2907. https://doi.org/10.1128/AEM.01530-08

Holdeman, L. V, Johnson, J.L., 1977. Bacteroides disiens sp. nov. and Bacteroides bivius sp. nov. from Human Clinical Infections. Int. J. Syst. Bacteriol. 27, 337-345. https://doi.org/10.1099/00207713-27-4-337

Jakobsson, T., Forsum, U., 2007. Lactobacillus iners: a marker of changes in the vaginal flora? J. Clin. Microbiol. 45, 3145. https://doi.org/10.1128/JCM.00558-07

Javed, A., Parvaiz, F., Manzoor, S., 2019. Bacterial vaginosis: an insight into the prevalence, alternative regimen treatments and it's associated resistance patterns. Microb. Pathog. 127, 21-30. https://doi.org/10.1016/j.micpath.2018.11.046

Krasnopolsky, V.N., Prilepskaya, V.N., Polatti, F., Zarochentseva, N. V, Bayramova, G.R., Caserini, M., Palmieri, R., 2013. Efficacy of Vitamin C vaginal tablets as prophylaxis for recurrent bacterial vaginosis: a randomised, double-blind, placebo-controlled clinical trial. $J$ Clin Med Res 5, 309-315. https://doi.org/10.4021/jocmr1489w

La Scola, B., Khelaifia, S., Lagier, J.C., Raoult, D., 2014. Aerobic culture of anaerobic bacteria using antioxidants: a preliminary report. Eur. J. Clin. Microbiol. Infect. Dis. 33, 1781-1783. 
389

390

391

392

393

394

395

396

397

398

399

400

401

402

403

404

405

406

407

408

409

410

411

412

413

414

415

416

417

418

419

https://doi.org/10.1007/s10096-014-2137-4

Machado, D., Palmeira-de-Oliveira, A., Cerca, N., 2015. Optimization of culture conditions for Gardnerella vaginalis biofilm formation. J. Microbiol. Methods 118, 143-146. https://doi.org/10.1016/j.mimet.2015.09.007

Marrazzo, J.M., 2011. Interpreting the epidemiology and natural history of bacterial vaginosis: are we still confused? Anaerobe 17, 186-190. https://doi.org/10.1038/jid.2014.371

Mirani, Z.A., Khan, M.N., Siddiqui, A., Khan, F., Aziz, M., Naz, S., Ahmed, A., Khan, S.I., 2018. Ascorbic acid augments colony spreading by reducing biofilm formation of methicillin-resistant Staphylococcus aureus. Iran. J. Basic Med. Sci. 21, 175-180. https://doi.org/10.22038/IJBMS.2018.20714.5398

Muzny, C.A., Taylor, C.M., Swords, W.E., Tamhane, A., Chattopadhyay, D., Cerca, N., Schwebke, J.R., 2019. An updated conceptual model on the pathogenesis of bacterial vaginosis. J. Infect. Dis. 220, 1399-1405. https://doi.org/10.1093/infdis/jiz342

Ng, J., Ng, L.-K., Chow, A.W., Dillon, J.-A.R., 1994. Identification of five Peptostreptococcus species isolated predominantly from the female genital tract by using the R rapid ID32A system. J. Clin. Microbiol. 32, 1302-1307.

Onderdonk, A.B., Delaney, M.L., Fichorova, N., 2016. The human microbiome during bacterial vaginosis. Clin. Microbiol. Rev. 29, 223-238. https://doi.org/10.1128/CMR.00075-15

Pandit, S., Ravikumar, V., Abdel-Haleem, A.M., Derouiche, A., Mokkapati, V.R.S.S., Sihlbom, C., Mineta, K., Gojobori, T., Gao, X., Westerlund, F., Mijakovic, I., 2017. Low concentrations of vitamin $\mathrm{C}$ reduce the synthesis of extracellular polymers and destabilize bacterial biofilms. Front. Microbiol. 8, 2599. https://doi.org/10.3389/fmicb.2017.02599

Patterson, J.L., Stull-Lane, A., Girerd, P.H., Jefferson, K.K., 2010. Analysis of adherence, biofilm formation and cytotoxicity suggests a greater virulence potential of Gardnerella vaginalis relative to other bacterial-vaginosis-associated anaerobes. Microbiology 156, 392399. https://doi.org/10.1099/mic.0.034280-0

Peeters, E., Nelis, H.J., Coenye, T., 2008. Comparison of multiple methods for quantification of microbial biofilms grown in microtiter plates. J. Microbiol. Methods 72, 157-165. https://doi.org/10.1016/j.mimet.2007.11.010

Petrova, M.I., Lievens, E., Malik, S., Imholz, N., Lebeer, S., 2015. Lactobacillus species as biomarkers and agents that can promote various aspects of vaginal health. Front. Physiol. 6, 
420

421

422

423

424

425

426

427

428

429

430

431

432

433

434

435

436

437

438

439

440

441

442

443

444

445

446

447

448

449

450

1-18. https://doi.org/10.3389/fphys.2015.00081

Polatti, F., Rampino, M., Magnani, P., Mascarucci, P., 2006. Vaginal pH-lowering effect of locally applied vitamin $\mathrm{C}$ in subjects with high vaginal pH. Gynecol. Endocrinol. 22, 230234. https://doi.org/10.1080/09513590600647441

Rampersaud, R., Planet, P.J., Randis, T.M., Kulkarni, R., Aguilar, J.L., Lehrer, R.I., Ratner, A.J., 2011. Inerolysin, a cholesterol-dependent cytolysin produced by Lactobacillus iners. J. Bacteriol. 193, 1034-1041. https://doi.org/10.1128/JB.00694-10

Ravel, J., Gajer, P., Abdo, Z., Schneider, G.M., Koenig, S.S.K., McCulle, S.L., Karlebach, S., Gorle, R., Russell, J., Tacket, C.O., Brotman, R.M., Davis, C.C., Ault, K., Peralta, L., Forney, L.J., 2011. Vaginal microbiome of reproductive-age women. Proc. Natl. Acad. Sci. 108, 4680-4687. https://doi.org/10.1073/pnas.1002611107

Ripolles-Avila, C., Hascoët, A.S., Guerrero-Navarro, A.E., Rodríguez-Jerez, J.J., 2018.

Establishment of incubation conditions to optimize the in vitro formation of mature Listeria monocytogenes biofilms on food-contact surfaces. Food Control 92, 240-248. https://doi.org/10.1016/j.foodcont.2018.04.054

Rodriguez Jovita, M., Collins, M.D., Sjodén, B., Falsen, E., 1999. Characterization of a novel Atopobium isolate from the human vagina: description of Atopobium vaginae sp. nov. Int. J. Syst. Bacteriol. 49, 1573-1576. https://doi.org/10.1099/00207713-49-4-1573

Schwebke, J.R., 2000. Bacterial vaginosis. Curr. Infect. Dis. Rep. 2, 14-17. https://doi.org/10.1007/s11908-000-0082-0

Spiegel, C.A., Roberts, M., 1984. Mobiluncus gen. nov., Mobiluncus curtisii subsp. curtisii sp. nov., Mobiluncus curtisii subsp. holmesii subsp. nov., and Mobiluncus mulieris sp. nov., curved rods from the human vagina. Int. J. Syst. Bacteriol. 34, 177-184. https://doi.org/10.1099/00207713-34-2-177

Srinivasan, S., Munch, M.M., Sizova, M. V., Fiedler, T.L., Kohler, C.M., Hoffman, N.G., Liu, C., Agnew, K.J., Marrazzo, J.M., Epstein, S.S., Fredricks, D.N., 2016. More easily cultivated than identified: classical isolation with molecular identification of vaginal bacteria. J. Infect. Dis. 214, S21-S28. https://doi.org/10.1093/infdis/jiw192

Stalons, D.R., Thornsberry, C., Dowell, V.R.J., 1974. Effect of culture medium and carbon dioxide concentration on growth of anaerobic bacteria commonly encountered in clinical specimens. Appl. Microbiol. 27, 1098-1104. https://doi.org/10.1128/aem.27.6.1098- 
451

452

453

454

455

456

457

458

459

460

461

462

463

464

465

466

467

468

469

470

471

472

473

474

475

476

477

478

479

480

1104.1974

Stingley, R.L., Liu, H., Mullis, L.B., Elkins, C.A., Hart, M.E., 2014. Staphylococcus aureus toxic shock syndrome toxin-1 (TSST-1) production and Lactobacillus species growth in a defined medium simulating vaginal secretions. J. Microbiol. Methods 106, 57-66. https://doi.org/10.1016/j.mimet.2014.08.002

Swidsinski, A., Loening-Baucke, V., Mendling, W., Dörffel, Y., Schilling, J., Halwani, Z., Jiang, X.F., Verstraelen, H., Swidsinski, S., 2014. Infection through structured polymicrobial Gardnerella biofilms (StPM-GB). Histol. Histopathol. 29, 567-587. https://doi.org/10.14670/HH-29.10.567

Swidsinski, A., Mendling, W., Loening-Baucke, V., Ladhoff, A., Swidsinski, S., Hale, L.P., Lochs, H., 2005. Adherent biofilms in bacterial vaginosis. Obstet. Gynecol. 106, 10131023. https://doi.org/10.1097/01.AOG.0000183594.45524.d2

Turovskiy, Y., Cheryian, T., Algburi, A., Wirawan, R.E., Takhistov, P., Sinko, P.J., Chikindas, M.L., 2012. Susceptibility of Gardnerella vaginalis biofilms to natural antimicrobials subtilosin, $\varepsilon$-poly-L-lysine, and lauramide arginine ethyl ester. Infect. Dis. Obstet. Gynecol. 2012, 1-9. https://doi.org/10.1155/2012/284762

Vaneechoutte, M., 2017. Lactobacillus iners, the unusual suspect. Res. Microbiol. 168, 826-836. https://doi.org/10.1016/j.resmic.2017.09.003

Vaneechoutte, M., Guschin, A., Van Simaey, L., Gansemans, Y., Van Nieuwerburgh, F., Cools, P., 2019. Emended description of Gardnerella vaginalis and description of Gardnerella leopoldii sp. nov., Gardnerella piotii sp. nov. and Gardnerella swidsinskii sp. nov., with delineation of 13 genomic species within the genus Gardnerella. Int. J. Syst. Evol. Microbiol. 69, 679-687. https://doi.org/10.1099/ijsem.0.003200

Weeks, R.M., Moretti, A., Song, S., Uhrich, K., Karlyshev, A. V., Chikindas, M.L., 2019. Cationic amphiphiles against Gardnerella vaginalis resistant strains and bacterial vaginosisassociated pathogens. Pathog. Dis. 77, pii: ftz059. https://doi.org/10.1093/femspd/ftz059

Wijesinghe, G., Dilhari, A., Gayani, B., Kottegoda, N., Samaranayake, L., Weerasekera, M., 2019. Influence of laboratory culture media on in vitro growth, adhesion, and biofilm formation of Pseudomonas aeruginosa and Staphylococcus aureus. Med. Princ. Pract. 28, 28-35. https://doi.org/10.1159/000494757 


\section{Table $\mathbf{1}$ (on next page)}

Cultivable bacterial species used for planktonic and biofilm growth assays.

${ }^{1}$ To determine how often the selected cultivable species have been reported with BV, a query in PubMed was performed by using a specific combination of keywords as "bacterial vaginosis" and "name of each species" (e.g. "Gardnerella" and "bacterial vaginosis"). We designated as "often described" those species referred in more than 50 articles in the last 25 years, while "commonly described" had at least 10 articles in the same period. Of note, the selected bacterial species used herein have been pointed out in several studies (Diop et al., 2019; Onderdonk et al., 2016; Ravel et al., 2011) as potential microbial pathogens involved in BV development. ${ }^{2}$ The partial $16 \mathrm{~S}$ ribosomal RNA gene sequences of Gardnerella sp. is downloadable from NCBI. UM: University of Minho, Portugal. In addition, the strain was phenotypically and genotypically characterized by Castro and colleagues ( Castro et al., 2020, 2018, 2015). Of note that Gardnerella sp. UM241 did not match with any of the Gardnerella species described by Vaneechoutte and colleagues (Vaneechoutte et al., 2019) (i.e. G. vaginalis, G. piotii, G. leopoldii and G. swidsinskii), belonging to a yet unidentified Gardnerella species ( Castro et al., 2020) . 


\section{Table 1.}

\section{Cultivable bacterial species used for planktonic and biofilm growth assays.}

\begin{tabular}{|c|c|c|c|}
\hline Species & Strain & Origin & Association with $\mathbf{B V}^{1}$ \\
\hline Gardnerella sp. & UM2412 & $\begin{array}{l}\text { Isolated from women diagnosed } \\
\text { with BV }\end{array}$ & Often described \\
\hline Atopobium vaginae & ATCC BAA-55 & $\begin{array}{l}\text { Isolated from vaginal microbiota of } \\
\text { a healthy woman (Rodriguez Jovita } \\
\text { et al., 1999) }\end{array}$ & Often described \\
\hline Lactobacillus iners & CCUG $28746^{\mathrm{T}}$ & $\begin{array}{l}\text { Isolated from human urine (Falsen } \\
\text { et al., 1999) }\end{array}$ & Commonly described \\
\hline Mobiluncus curtisii & ATCC $35241^{\mathrm{T}}$ & $\begin{array}{l}\text { Isolated from women with BV } \\
\text { (Spiegel and Roberts, 1984) }\end{array}$ & Commonly described \\
\hline Peptostreptococcus anaerobius & ATCC $27337^{\mathrm{T}}$ & $\begin{array}{l}\text { Isolated from female genital tract } \\
\text { (Ng et al., 1994) }\end{array}$ & Commonly described \\
\hline Prevotella bivia & ATCC $29303^{\mathrm{T}}$ & $\begin{array}{l}\text { Isolated from endometrium } \\
\text { (Holdeman and Johnson, 1977) }\end{array}$ & Commonly described \\
\hline
\end{tabular}

$3^{1}$ To determine how often the selected cultivable species have been reported with BV, a query in PubMed was

4 performed by using a specific combination of keywords as "bacterial vaginosis" and "name of each species" (e.g.

5 "Gardnerella" and "bacterial vaginosis"). We designated as "often described" those species referred in more than 50

6 articles in the last 25 years, while "commonly described" had at least 10 articles in the same period. Of note, the

7 selected bacterial species used herein have been pointed out in several studies (Diop et al., 2019; Onderdonk et al.,

8 2016; Ravel et al., 2011) as potential microbial pathogens involved in BV development.

$9{ }^{2}$ The partial 16S ribosomal RNA gene sequences of Gardnerella sp. is downloadable from NCBI. UM: University of

10 Minho, Portugal. In addition, the strain was phenotypically and genotypically characterized by Castro and colleagues

11 (Castro et al., 2020, 2018, 2015). Of note that Gardnerella sp. UM241 did not match with any of the Gardnerella

12 species described by Vaneechoutte and colleagues (Vaneechoutte et al., 2019) (i.e. G. vaginalis, G. piotii, G. leopoldii

13 and G. swidsinskii), belonging to a yet unidentified Gardnerella species (Castro et al., 2020). 


\section{Table 2 (on next page)}

Culture media used for the growth of BV-associated anaerobes.

${ }^{1}$ The supplementation of the culture media with $0.1 \%(w / v)$ L-ascorbic acid was designated with the abbreviation "Aa", added at the end of each medium' s name as follows, e.g. sBHI supplemented with Lascorbic acid became sBHI.Aa. The other three culture media were abbreviated by following the same rule.

${ }^{2}$ The mGTS medium has already $0.1 \%(w / v)$ L-ascorbic acid in its composition. 
1 Table 2.

2 Culture media used for the growth of BV-associated anaerobes.

\begin{tabular}{|c|c|c|c|}
\hline Culture medium & Composition & Supplementation & Abbreviation \\
\hline $\begin{array}{l}\text { Brain heart } \\
\text { infusion broth } \\
\text { (Liofilchem, Italy) }\end{array}$ & As described by the manufacturer & $\begin{array}{l}2 \%(\mathrm{w} / \mathrm{w}) \text { Gelatine (Liofilchem, Italy); } \\
0.1 \%(\mathrm{w} / \mathrm{w}) \text { Starch (Panreac, Spain); } \\
0.5 \%(\mathrm{w} / \mathrm{w}) \text { Yeast extract (Liofilchem, } \\
\text { Italy) }\end{array}$ & sBHI/ sBHI.Aa ${ }^{1}$ \\
\hline $\begin{array}{l}\text { Brucella broth } \\
\text { (Liofilchem, Italy) }\end{array}$ & As described by the manufacturer & $\begin{array}{l}0.0005 \%(\mathrm{w} / \mathrm{v}) \text { Hemin (Sigma, China); } \\
0.0001 \%(\mathrm{w} / \mathrm{v}) \text { Vitamin } \mathrm{K}_{1} \text { (Sigma, } \\
\text { China) }\end{array}$ & BHV/ BHV.Aa ${ }^{1}$ \\
\hline $\begin{array}{l}\text { New York City III } \\
\text { broth }\end{array}$ & $\begin{array}{l}1.5 \%(\mathrm{w} / \mathrm{v}) \text { Bacto proteose peptone no. } 3(\mathrm{BD}, \text { France); } \\
0.5 \%(\mathrm{w} / \mathrm{v}) \text { Glucose (Fisher Scientific, UK); } \\
0.24 \%(\mathrm{w} / \mathrm{v}) \mathrm{HEPES}(\mathrm{VWR}, \mathrm{USA}) ; \\
0.5 \%(\mathrm{w} / \mathrm{v}) \mathrm{NaCl}(\mathrm{VWR}, \mathrm{USA}) ; \\
0.38 \%(\mathrm{w} / \mathrm{v}) \text { Yeast extract (Liofilchem, Italy) }\end{array}$ & $\begin{array}{l}10 \%(\mathrm{v} / \mathrm{v}) \text { Inactivated horse serum } \\
\text { (Biowest, France) }\end{array}$ & NYC/ NYC.Aa ${ }^{1}$ \\
\hline $\begin{array}{l}\text { Schaedler broth } \\
\text { (Liofilchem, Italy) }\end{array}$ & As described by the manufacturer & - & SB/ SB.Aa ${ }^{1}$ \\
\hline $\begin{array}{l}\text { Chemically } \\
\text { defined medium } \\
\text { simulating genital } \\
\text { tract secretions } \\
\text { (Stingley et al., } \\
\text { 2014) }\end{array}$ & $\begin{array}{l}\text { Part I: } 0.35 \% \mathrm{NaCl} ; 0.15 \% \mathrm{KCl} ; 0.174 \% \mathrm{~K}_{2} \mathrm{HPO}_{4} ; 0.136 \% \mathrm{KH}_{2} \mathrm{PO}_{4} ; \\
1.08 \% \text { glucose; } 0.05 \% \text { cysteine } \mathrm{HCl} \text {. } \\
\text { Part II: } 0.1 \% \text { glycogen; } 0.03 \% \text { mucin; } 0.02 \% \text { tween } 20 ; 0.05 \% \text { urea; } \\
0.0005 \% \text { hemin; } 0.0001 \% \text { vitamin } \mathrm{K}_{1} ; 0.2 \% \text { bovine serum albumin; } \\
0.03 \% \mathrm{MgSO} \text {; } 0.004 \% \mathrm{NaHCO}_{3} ; 0.1 \% \text { sodium acetate; } 0.005 \% \mathrm{MnCl}_{2} \text {. } \\
\text { Part III: } 0.0005 \% \text { biotin; } 5.0 \% \text { myo-inositol; } 0.05 \% \text { niacinamide; } 0.05 \% \\
\text { pyridoxine } \mathrm{HCI} ; 0.05 \% \text { thiamine } \mathrm{HCI} ; 0.05 \% \text { D-calcium pantothenate; } \\
0.05 \% \text { folic acid; } 0.001 \% \text { p-aminobenzoic acid; } 0.05 \% \text { choline chloride; } \\
0.01 \% \text { riboflavin; } 0.1 \% \text { L-ascorbic acid; } 0.0005 \% \text { vitamin A (retinol); } \\
0.0005 \% \text { vitamin D (cholecalciferol); } 0.001 \% \text { vitamin } \mathrm{B}_{12} . \\
\text { Part IV (amino acids): } 0.032 \% \text { alanine; } 0.008 \% \text { arginine; } 0.076 \% \text { aspartic } \\
\text { acid; } 0.036 \% \text { glutamic acid; } 0.04 \% \text { glutamine; } 0.02 \% \text { glycine; } 0.016 \% \\
\text { histidine; } 0.012 \% \text { isoleucine; } 0.02 \% \text { leucine; } 0.02 \% \text { lysine; } 0.004 \% \\
\text { methionine; } 0.004 \% \text { phenylalanine; } 0.028 \% \text { proline; } 0.012 \% \text { serine; } \\
0.012 \% \text { threonine; } 0.004 \% \text { tryptophan; } 0.02 \% \text { tyrosine; } 0.068 \% \text { valine. } \\
\text { Part V (UPI): } 0.05 \% \text { uracil; } 0.01 \% \text { sodium pyruvate; } 0.02 \% \text { inosine. }\end{array}$ & - & mGTS $^{2}$ \\
\hline
\end{tabular}


$3{ }^{1}$ The supplementation of the culture media with $0.1 \%(\mathrm{w} / \mathrm{v})$ L-ascorbic acid was designated with the abbreviation "Aa", added at the end of each medium's name 4 as follows, e.g. sBHI supplemented with L-ascorbic acid became sBHI.Aa. The other three culture media were abbreviated by following the same rule.

$5 \quad{ }^{2}$ The mGTS medium has already $0.1 \%(w / v)$ L-ascorbic acid in its composition. 


\section{Figure 1}

Fold change in planktonic growth of BV-associated bacteria in the nine different culture media relative to $\mathrm{OD}_{620 \mathrm{~nm}}$ values measured at TOh.

Results represent the average \pm the standard deviation of at least three independent experiments. Statistical analysis was performed using one-way ANOVA and Dunnett's multiple comparisons test. Significant differences between NYC medium (our culture medium of choice) and other culture media are depicted with $* p<0.05$ and $* * p<0.01$. Other statistical analysis is shown in Table S1. 

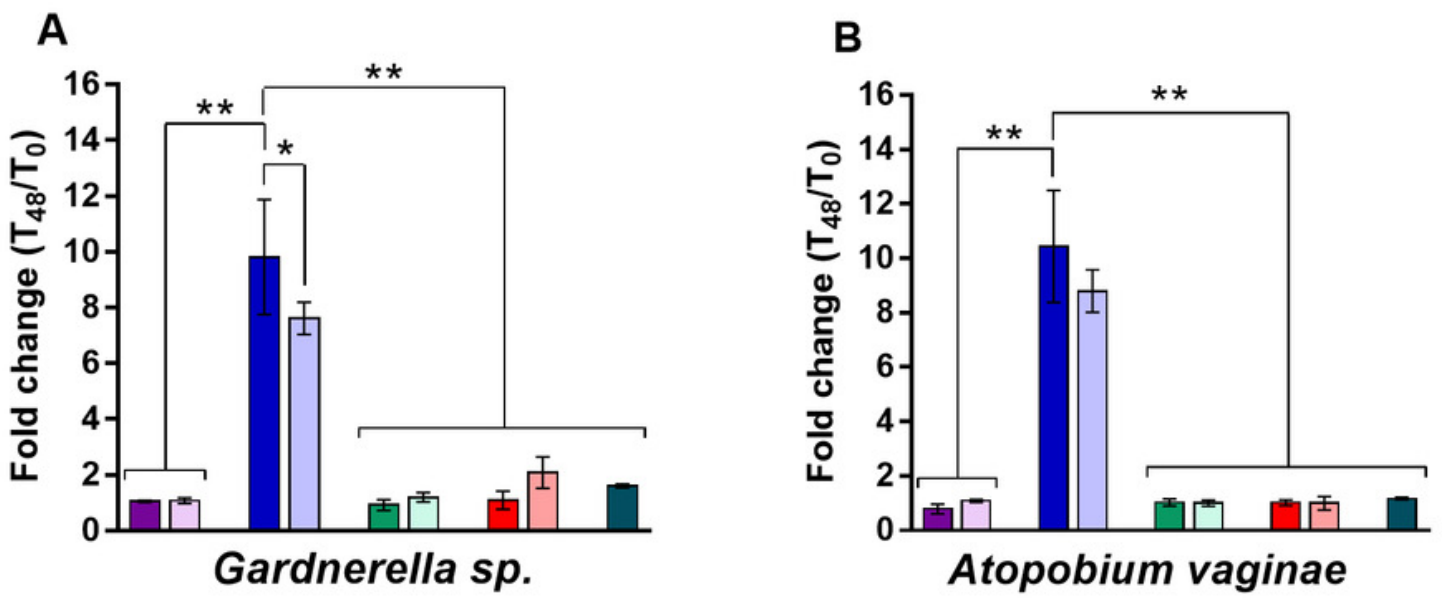

Culture medium

C
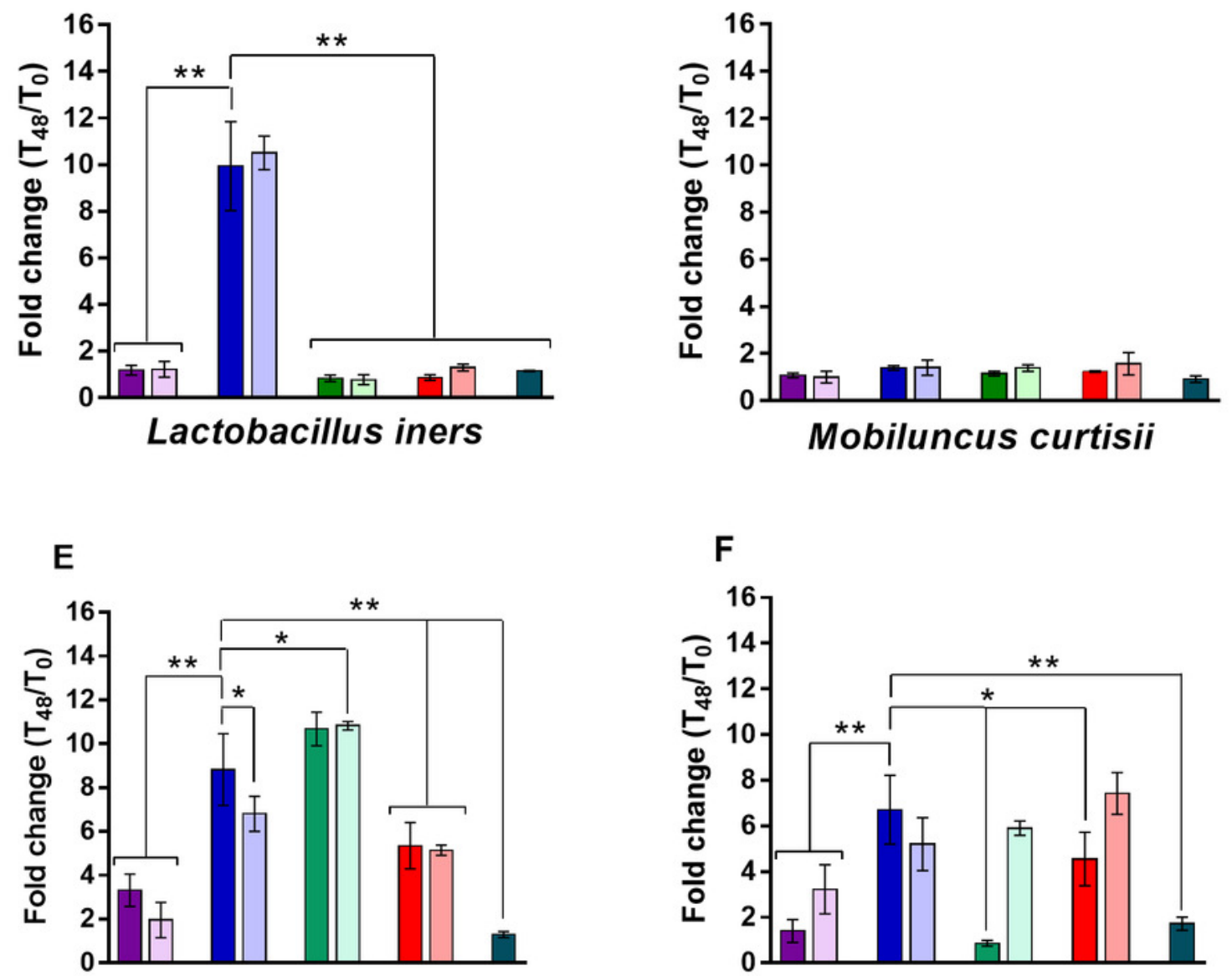

Peptostreptococcus anaerobius

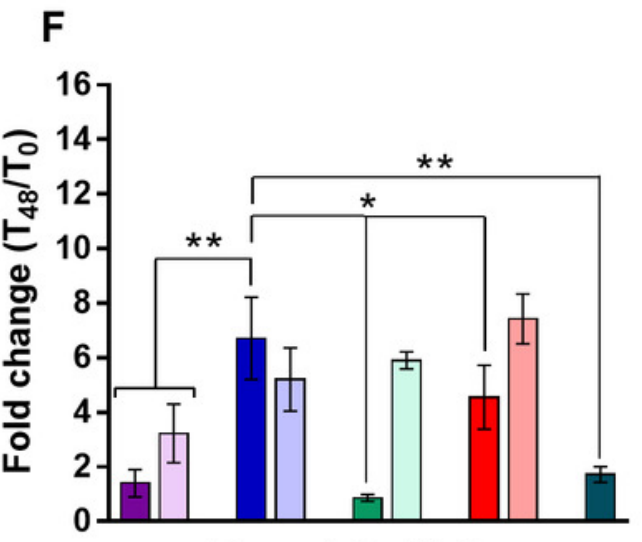

Prevotella bivia 


\section{Figure 2}

Biofilm formation of BV-associated bacteria in the nine different culture media over a 72 $h$ period.

Biofilm biomass was quantified using the crystal violet staining assay. Results represent the average \pm the standard deviation of at least three independent experiments performed with eight technical replicates. Statistical analysis was performed using one-way ANOVA and Dunnett's multiple comparisons test. Significant differences between biofilm biomass formed in NYC medium (our culture medium of choice) and other culture media are represented with ${ }^{*} p<0.05$ and ${ }^{* *} p<0.01$. Other statistical analysis is shown in Table $S 2$. 
A

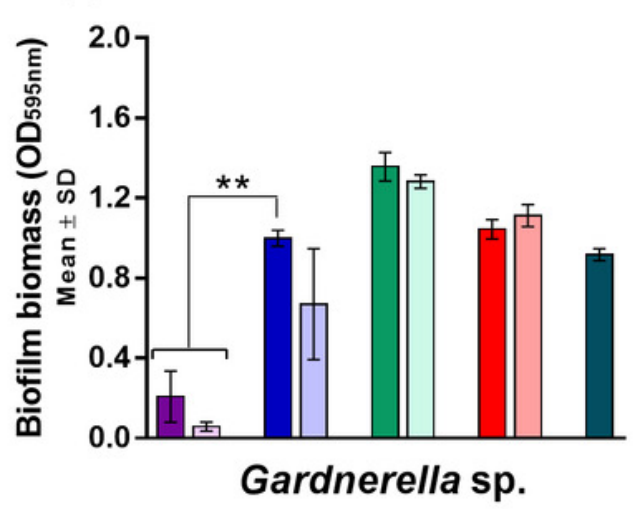

C

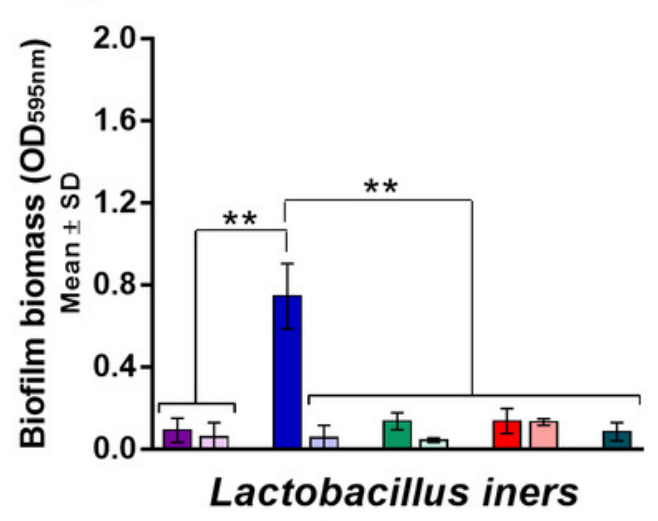

E

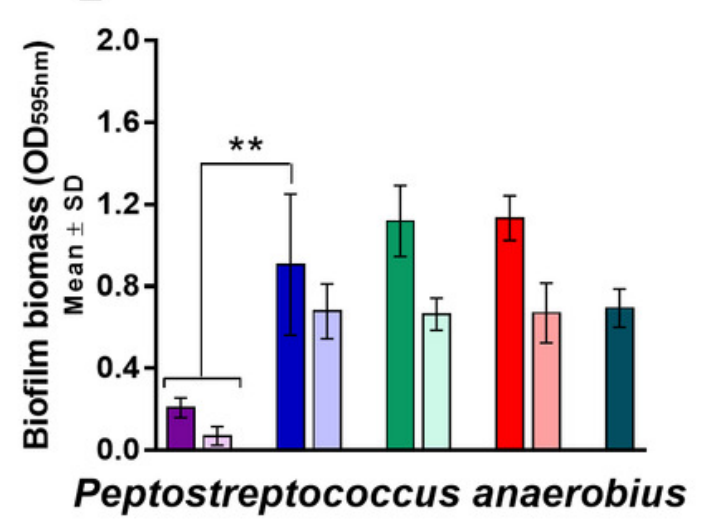

B

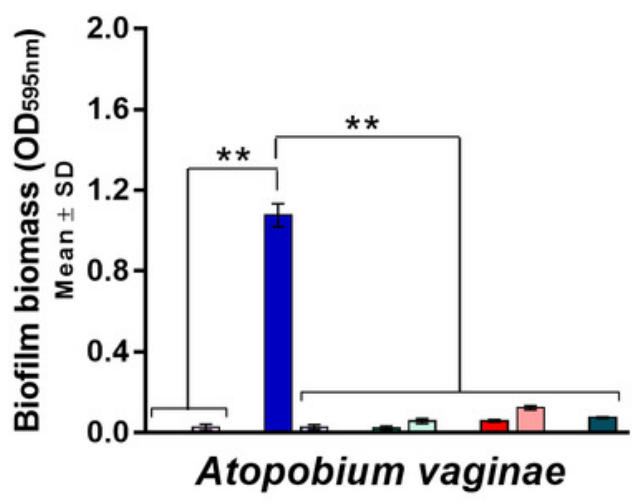

D

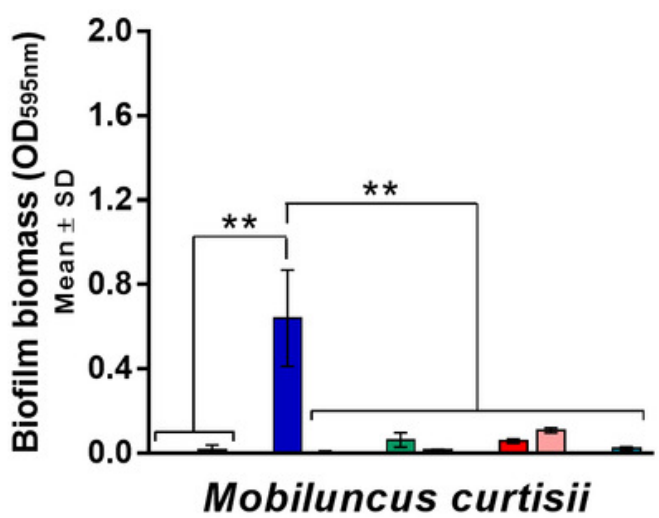

F

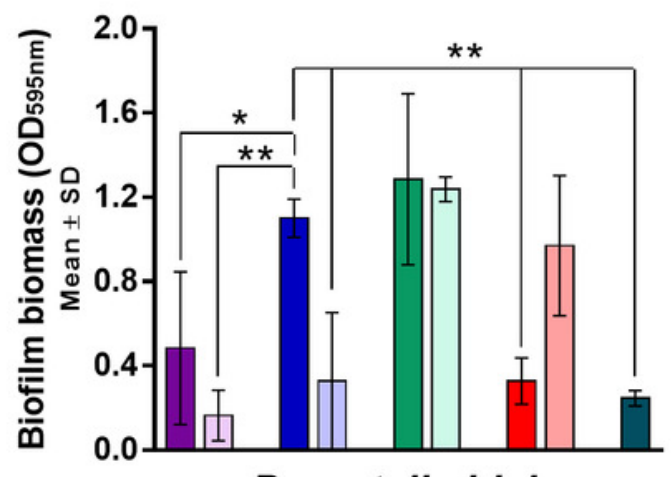

Culture medium

BHV

$\square$ BHV.Aa

- NYC

$\square$ NYC.Aa

$\square$ SB

$\square$ SB.Aa

sBHI

$\square$ sBHI.Aa

- mGTS

\section{Prevotella bivia}

